\title{
SUBSTÂNCIAS ANTIFÚNGICAS DE Xylaria sp., UM FUNGO ENDOFÍTICO ISOLADO DE Palicourea marcgravii (RUBIACEAE)
}

\author{
Mariana C. Cafêu, Geraldo H. Silva, Helder L. Teles, Vanderlan da S. Bolzani e Ângela R. Araújo* \\ Instituto de Química, Universidade Estadual Paulista, CP 355, 14801-970 Araraquara - SP \\ Maria Cláudia M. Young \\ Seção de Fisiologia e Bioquímica de Plantas, Instituto de Botânica, CP 4005, 01061-970 São Paulo - SP \\ Ludwig H. Pfenning \\ Departamento de Fitopatologia, Universidade Federal de Lavras, 37200-000 Lavras - MG
}

Recebido em 31/8/04; aceito em 11/3/05; publicado na web em 10/8/05

\begin{abstract}
ANTIFUNGAL COMPOUNDS OF Xylaria sp., AN ENDOPHYTIC FUNGUS ISOLATED FROM Palicourea marcgravii (RUBIACEAE). Five compounds, 2-hexyl-3-methyl-butanodioic acid (1), cytochalasin D (2), 7-dechlorogriseofulvin (3), cytochalasin B (4) and griseofulvin (5), have been isolated from the endophytic fungus Xylaria sp., and their structures were elucidated on the basis of spectroscopic data. In the bioautography assay against Cladosporium cladosporioides and Cladosporium sphaerospermum, compounds 1 and 2 were found to be active while compounds 3, 4 and 5 did not show antifungal activity.
\end{abstract}

Keywords: endophytic fungus; Xylaria sp.; antifungal compounds.

\section{INTRODUÇÃO}

Vários produtos naturais com propriedades terapêuticas foram isolados de espécies de fungos, especialmente agentes quimioterápicos, como penicilinas e cefalosporinas usadas até hoje como antibióticos; mevinolina, um agente redutor de colesterol; bleomicinas, daunorubicinas e análogos, como agentes antitumorais; avermectina B, um anti-helmíntico de largo espectro, entre outros ${ }^{1,2}$. Recentemente, o aumento de infecções fúngicas causadas por fungos oportunistas, como Candida e Aspergillus, e a resistência microbiana a uma série de antibióticos disponível no mercado estão motivando, novamente, as companhias farmacêuticas a investirem na busca por substâncias antifúngicas de fontes naturais² ${ }^{2}$.

Dentre os microrganismos que acumulam substâncias antifúngicas, fungos e leveduras destacam-se pela quantidade de produtos farmacêuticos de uso corrente na medicina com tais propriedades.

Os fungos filamentosos, onde os endofíticos estão incluídos, constituem um grupo de microrganismos que biossintetizam uma quantidade fantástica de metabólitos secundários, chegando, em casos especiais, a uma produção $73 \%$ superior a de outras classes de microrganismos ${ }^{3}$. Fungos endofíticos são microrganismos que habitam o interior de plantas e constituem-se em uma fonte valiosa de produtos bioativos ${ }^{4}$. Este nicho de fungos associados a espécies vegetais de Cerrado e da Mata Atlântica permanece praticamente sem qualquer estudo químico e biológico sendo, portanto, uma fonte potencial de substâncias de interesse farmacológico.

Dando continuidade às nossas pesquisas de bioprospecção, várias espécies vegetais de Cerrado e Mata Atlântica foram selecionadas para isolamento e cultivo de fungos endofíticos. De Palicourea marcgravii St. Hil., uma Rubiaceae de larga ocorrência no Cerrado, e conhecida popularmente como "erva de rato" ou "café bravo", foram isolados vários fungos endofíticos. Dentre os já identificados, Xylaria sp. foi selecionado para estudo devido a atividade apresentada por seu extrato bruto frente aos fungos fitopatogênicos

*e-mail: araujoar@iq.unesp.br
Cladosporium cladosporioides e Cladosporium sphaerospermum, indicando a produção de metabólitos com atividade antifúngica em potencial. Xylaria sp. foi submetido aos procedimentos usuais de cultivo em larga escala para fornecimento dos extratos brutos que após fracionamento bioguiado, levou ao isolamento de 5 substâncias: ácido 2-hexilideno-3-metilbutanodióico (1), citocalasina D (2), 7-declorogriseofulvina (3), citocalasina B (4) e griseofulvina (5) (Figura 1).

\section{PARTE EXPERIMENTAL}

\section{Procedimentos experimentais gerais}

Os espectros de $\mathrm{RMN}$ de ${ }^{1} \mathrm{He}{ }^{13} \mathrm{C}$ e experimentos bidimensionais foram realizados em espectrômetro Varian INOVA-500 operando a $500 \mathrm{MHz}$ para o núcleo de ${ }^{1} \mathrm{H}$ e em $125 \mathrm{MHz}$ para o núcleo de ${ }^{13} \mathrm{C}$; TMS foi utilizado como referência interna. Os espectros de massas foram registrados em espectrômetro de massas de baixa resolução FISONS- Modelo VG Platfform II, no modo Electrospray. As análises cromatográficas por CLAE no modo analítico foram realizadas em equipamento ProStar da Varian acoplado ao detetor ProStar 330 de arranjos de diodos, utilizando coluna analítica Phenomenex Fenil-Hexil ( 250 x 4,60 mm; $5 \mu \mathrm{m})$. No modo preparativo, as análises por CLAE foram realizadas em equipamento ProStar da Varian acoplado ao detetor ProStar UV, utilizando coluna Phenomenex Fenil-Hexil (250 x 21,20 mm; $10 \mu \mathrm{m})$. Nas separações cromatográficas em coluna aberta ou sob pressão foram utilizadas sílica gel C-18 (Merck) e Sephadex LH-20 (Merck) como fases estacionárias. Para cultivo de Xylaria sp. em meio líquido foram utilizados 20 frascos de Erlenmeyers $(500 \mathrm{~mL})$ contendo $200 \mathrm{~mL}$ de meio de cultivo preparado a partir de água MiliQ e PDB (DIFCO), posteriormente submetidos a esterilização em autoclave a $121{ }^{\circ} \mathrm{C}$ e $1 \mathrm{~atm}$ de pressão por $20 \mathrm{~min}$. Para cultivo em meio sólido de milho foram colocados $90 \mathrm{~g}$ de milho descascado (Yoki) em 2 Erlenmeyers $(500 \mathrm{~mL})$ contendo $75 \mathrm{~mL}$ de água destilada. Estes meios foram autoclavados quatro vezes (4 dias) por 40 min à temperatura de $121^{\circ} \mathrm{C}$ e $1 \mathrm{~atm}$ de pressão. 
<smiles>CCCCCC=C(C(=O)O)C(C)C(=O)O</smiles>

1

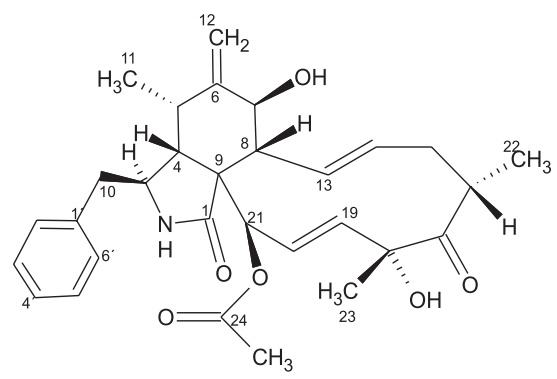

2

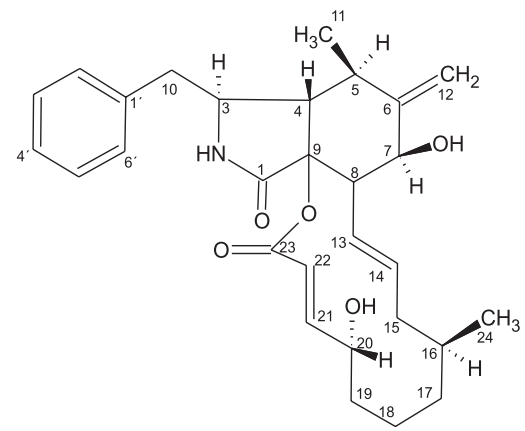

4<smiles>[R]c1c(OC)c([2H])c(OC)c2c1O[C@]1(C(=O)OC)C(OC)=CC(=O)C[C@]21C</smiles>

$3 \mathrm{R}=\mathrm{H}$

$5 \mathrm{R}=\mathrm{Cl}$

Figura 1. Substâncias produzidas por Xylaria sp.

\section{Material vegetal}

A espécie vegetal Palicourea marcgravii St. Hil. (Rubiaceae) foi coletada, em junho de 2001, na Estação Ecológica Experimental de Mogi-Guaçú (SP). Uma exsicata do material vegetal identificado encontra-se depositada no Herbário do Instituto de Botânica da Secretaria do Meio Ambiente de São Paulo (SP).

\section{Isolamento da cepa fúngica}

O fungo endofítico foi isolado de folhas saudáveis de Palicourea marcgravii. As folhas foram lavadas com água e sabão e esterilizadas por imersão em $\mathrm{NaClO}$ 1\% (5 min) e em etanol 70\% (1 min), seguidas de lavagem com água estéril (10 min) e secagem ${ }^{5}$. Após o processo de esterilização, as folhas foram cortadas e incubadas em placas de Petri contendo PDA, às quais foi adicionado o antibióti- co sulfato de antamicina $(50 \mathrm{mg} / \mathrm{mL})$ para evitar crescimento de bactérias contaminantes. Repiques sucessivos em placas de Petri contendo PDA resultaram no isolamento de 4 linhagens codificadas como PM-01, PM-02, PM-03 e PM-04. Estas linhagens foram preservadas em frascos esterilizados com água. A linhagem PM03, identificada como Xylaria sp. pelo Prof. Dr. L. H. Pfenning, Departamento de Fitopatologia da Universidade Federal de Lavras - MG, foi selecionada para estudo químico, visando a obtenção das substâncias bioativas.

\section{Cultivo do microrganismo e obtenção dos extratos brutos}

A cepa fúngica de Xylaria sp. foi repicada em placa de Petri contendo PDA e incubada por 7 dias. Após este período, o fungo foi inoculado nos meios de cultivo líquido (PDB) e sólido (Milho). Em PDB, Xylaria sp. foi cultivada em $4000 \mathrm{~mL}$ de meio (20 frascos de Erlenmeyers de $500 \mathrm{~mL}$ ) e mantida em incubadora rotatória ("shaker") sob temperatura controlada $\left(24^{\circ} \mathrm{C}\right)$ por aproximadamente 28 dias, fornecendo o caldo fermentado. $\mathrm{O}$ caldo foi submetido à filtração para separação dos micélios e à partição líquido/líquido com AcOEt que, após a evaporação do solvente, forneceu 2,0 g do extrato bruto acetato de etila (PDB-AcOEt). Xylaria sp. também foi cultivada em meio sólido milho, onde permaneceu incubada por 21 dias, à $24{ }^{\circ} \mathrm{C}$ em modo estático. Em seguida, foi realizada a extração com metanol, filtração para separação dos micélios e concentração do solvente, fornecendo 5,3 g de extrato bruto metanólico (milho-MeOH).

\section{Isolamento dos constituintes químicos}

O extrato bruto PDB-AcOEt $(2,0 \mathrm{~g})$ foi dissolvido em acetato de etila e, em seguida, particionado com hexano e acetonitrila, resultando em duas soluções. A solução ACN, após concentração, forneceu a fração $\mathrm{ACN}(0,7 \mathrm{~g})$ que foi submetida à cromatografia em coluna usando sílica de fase reversa (C-18), eluída com MeOH: $\mathrm{H}_{2} \mathrm{O}$ no modo gradiente, resultando em sete sub-frações. As sub-frações ACN-4 a ACN-7 foram reunidas (ACN4-7; 400 mg) devido à bioatividade apresentada frente às linhagens Cladosporium cladosporioides e C. sphaerospermum. Esta sub-fração ACN4-7 foi submetida a uma partição com acetato de etila e solução de hidróxido de sódio 5\%. A fase aquosa foi tratada com ácido clorídrico até pH 5 e, em seguida, extraída com clorofórmio que, após a concentração do solvente, forneceu a substância $1(100 \mathrm{mg})$. A fase orgânica (AcOEt), após concentração do solvente, foi fracionada em uma coluna Sephadex LH-20 eluída com metanol, resultando em 10 subfrações (FCS 1-10). A sub-fração FCS-1 (23 mg) foi submetida à CLAE preparativa empregando modo de eluição isocrático acetonitrila:água (38:62), fluxo $20 \mathrm{~mL} / \mathrm{min}, \lambda 221 \mathrm{~nm}$ e coluna fenil hexil, resultando no isolamento da substância $2(3,5 \mathrm{mg})$.

$\mathrm{O}$ extrato bruto milho-MeOH (5,3 g) foi analisado por RMN de ${ }^{1} \mathrm{H}$, onde constataram-se vários sinais referentes a hidrogênios metínicos e metílicos típicos de açúcares. Para remover estes açúcares, uma porção do extrato ( $1 \mathrm{~g}$ ) foi dissolvida (parcialmente) em acetato de etila, obtendo-se a fração AcOEt-milho $(210 \mathrm{mg})$. Em seguida, esta fração foi submetida à cromatografia em coluna com sílica de fase reversa C-18 no modo de eluição isocrático empregando MeOH: $\mathrm{H}_{2} \mathrm{O}$ (80:20), resultando em M-1 (100 mg). A subfração M-1 foi purificada por CLAE preparativa utilizando como eluente $\mathrm{MeOH}: \mathrm{H}_{2} \mathrm{O}$ (60:40), fluxo de $15 \mathrm{~mL} / \mathrm{min}$, coluna fenil hexil e $\lambda 221 \mathrm{~nm}$ fornecendo 8 sub-frações (M1.1 - M1.8). As sub-frações M1.3, M1.5 e M1.7 resultaram no isolamento das substâncias 3 (8,3 mg), 4 (20,6 mg) e $\mathbf{5}(8,8 \mathrm{mg})$. 


\section{Bioensaio}

Os extratos brutos (PDB-AcOEt e milho-MeOH) e as substâncias isoladas foram submetidos à técnica de Bioautografia para detecção de atividade antifúngica. Este bioensaio consiste na nebulização de suspensões dos fungos Cladosporium cladosporioides e Cladosporium sphaerospermum (concentração $5 \times 10^{7}$ esporos $/ \mathrm{mL}$ em solução de glicose e sais) em placas de CCDC contendo $400 \mu \mathrm{g}$ dos extratos brutos e $100 \mu \mathrm{g}$ das substâncias puras, previamente eluídas com solvente adequado. Em seguida, as placas foram incubadas em câmara úmida a $25^{\circ} \mathrm{C}$ por $48 \mathrm{~h}$. O aparecimento de zonas de inibição na placa nebulizada com $C$. sphaerospermum e/ou C. cladosporioides indica a presença de substância(s) com potencial antifúngico ${ }^{6}$. A nistatina foi a substância utilizada como antifúngico padrão.

As substâncias puras que se mostraram potencialmente bioativas foram submetidas a bioensaio, para determinação do limite de detecção. Os resultados são expressos como valores de concentração da amostra $(\mu \mathrm{g} / \mathrm{mL})$ requeridos para produzir um halo de inibição comparável com o produzido pelo padrão nistatina. Os ensaios foram realizados em diferentes concentrações $(100,50,25,10,5$ e $1 \mu \mathrm{g} / \mathrm{mL}$ ) para as substâncias puras e para o padrão.

\section{RESULTADOS E DISCUSSÃO}

O estudo químico dos extratos brutos PDB-AcOEt e milho$\mathrm{MeOH}$, produzidos pelo fungo endofítico Xylaria sp., resultou no isolamento do ácido 2-hexilideno-3-metilbutanodióico (1), citocalasina D (2), 7-declorogriseofulvina (3), citocalasina B (4) e griseofulvina (5). A identificação destas substâncias foi realizada com base na comparação de seus dados espectroscópicos (RMN ${ }^{1} \mathrm{H}$ e ${ }^{13} \mathrm{C}, g \mathrm{COSY}, g \mathrm{HMQC}, g \mathrm{HMBC}$ e EM) com valores disponíveis na literatura. Os metabólitos 1-5 já haviam sido produzidos por fungos da família Xylariaceae, entretanto, este é o primeiro relato da produção de $\mathbf{3}, \mathbf{4}$ e $\mathbf{5}$ por um fungo endofítico.

A análise detalhada dos espectros de massas, RMN de ${ }^{1} \mathrm{H}$ e ${ }^{13} \mathrm{C}$ (uni e bidimensionais), além da comparação com dados da literatura $^{7}$, permitiu a identificação de $\mathbf{1}$ como ácido 2-hexilideno-3metilbutanodióico.

A substância 2 foi identificada como citocalasina $\mathrm{D}\left(\mathrm{C}_{30} \mathrm{H}_{37} \mathrm{NO}_{6}\right)$ através da análise dos dados de RMN de ${ }^{1} \mathrm{H}$ e ${ }^{13} \mathrm{C}$ e EM-ES $(+120$ V) e evidenciou 13 graus de insaturação. A análise dos dados de $\mathrm{RMN}$ de ${ }^{1} \mathrm{H}$ e ${ }^{13} \mathrm{C}$ indicou a presença de três carbonilas, um anel benzênico monossubstituído e seis carbonos olefínicos, o que resultou no grau de insaturação 10. Os 3 graus de insaturações remanescentes sugeriram que a molécula fosse constituída por 3 anéis, além do anel aromático. $\mathrm{O}$ espectro de hidrogênio apresentou sinais em $\delta_{\mathrm{H}}$ 3,57 e 5,26 característicos de hidrogênios carbinólicos, que foram atribuídos à H-7 e H-21. A geometria das ligações duplas $\Delta^{13-14}$ e $\Delta^{19-20}$ foi determinada como $E$, devido às constantes de acoplamento $(J=15,0 \mathrm{~Hz})$ entre os hidrogênios. Os sinais $\delta_{\mathrm{H}} 1,38$, $1,05,0,39$ e 2,27 foram atribuídos às metilas $\mathrm{H}_{3} \mathrm{C}-23, \mathrm{H}_{3} \mathrm{C}-22, \mathrm{H}_{3} \mathrm{C}$ $11 \mathrm{e}_{3} \mathrm{C}-25$, respectivamente. $\mathrm{O}$ sinal referente a uma metila em $\delta_{\mathrm{H}} 2,27$ é típico de citocalasina com um grupo acetato na forma de radical. Estas informações aliadas à análise dos dados espectrométricos de RMN de ${ }^{1} \mathrm{H},{ }^{13} \mathrm{C}$, gHMQC, $g \mathrm{HMBC}, g \mathrm{COSY}$, EM e comparação com os dados da literatura ${ }^{8}$ confirmaram a substância 2 como sendo uma citocalasina do tipo D. A configuração relativa de $\mathbf{2}$ foi determinada por comparação dos valores de deslocamentos químicos de $\mathrm{RMN}{ }^{1} \mathrm{H} \mathrm{e}{ }^{13} \mathrm{C}$, constantes de acoplamentos e comparação com os dados da literatura. Experimento de rotação ótica forneceu $[\alpha]_{\mathrm{D}}^{25}+15,4$ (c. $\left.0,25 \mathrm{EtOH}\right)$, condizente com a literatura $^{9}$, permitindo identificá-la como $(+)$-citocalasina D. Os valo- res dos deslocamentos químicos da citocalasina D (2) estão apresentados na Tabela 1.

A substância 4 foi identificada como citocalasina B através da análise dos dados espectrométricos de $\mathrm{RMN}$ de ${ }^{1} \mathrm{H}$ e ${ }^{13} \mathrm{C}$ (uni e bidimensionais), EM-ES (+120 V) e comparação com dados da literatura ${ }^{10,11}$. Os sinais registrados pelo espectro de RMN de ${ }^{1} \mathrm{H}$ em $\delta_{H} 5,81,5,03,6,76$ e 5,70 foram atribuídos aos hidrogênios das ligações duplas $\Delta^{13-14}$ e $\Delta^{21-22}$, respectivamente. Ambas ligações duplas possuem geometria $E$, uma vez que a constante de acoplamento entre os hidrogênios H-13-H-14 e H-21-H-22 é de 15,5 Hz. Pelo espectro de $\mathrm{RMN}$ de ${ }^{1} \mathrm{H}$ também foi possível verificar a presença de 2 metilas $\left(\mathrm{CH}_{3}-11\right.$ e $\left.\mathrm{CH}_{3}-24\right)$ e de hidrogênios carbinólicos $\left(\delta_{\mathrm{H}}\right.$ 3,61 e 4,36) atribuídos aos hidrogênios H-7 e H-20. Os dados espectrométricos de $\mathbf{4}$ foram comparados com os da citocalasina D (2), onde foi possível notar uma grande semelhança nos sinais e constatou-se que $\mathbf{4}$ se tratava de uma substância da classe de citocalasinas. A diferença marcante entre $\mathbf{2}$ e $\mathbf{4}$ está na ausência de um sinal entre $\delta_{\mathrm{H}} 2,10-2,30$, atribuído ao deslocamento químico da metila do acetato, o que permitiu propor que a ligação com o C-9 ocorre com o oxigênio do grupo acetato, em vez de se ligar no átomo de carbono como no caso da citocalasina D (2). A configuração relativa de $\mathbf{4}$ foi determinada por comparação dos valores de

Tabela 1. Dados de RMN de ${ }^{1} \mathrm{H}^{*}(500 \mathrm{MHz})$ e ${ }^{13} \mathrm{C}(125 \mathrm{MHz}) \mathrm{da}$ citocalasina D (2) e citocalasina B (4) em DMSO-d 6 , $\delta$ (ppm)

\begin{tabular}{|c|c|c|c|c|}
\hline \multirow{2}{*}{ Posiçã } & \multicolumn{2}{|l|}{2} & \multicolumn{2}{|l|}{4} \\
\hline & $\delta{ }^{1} \mathrm{H}$ & $\delta{ }^{13} \mathrm{C}$ & $\delta{ }^{1} \mathrm{H}$ & $\delta^{13} \mathrm{C}$ \\
\hline 1 & - & 173,6 & - & 170,7 \\
\hline 2 & 8,03 & - & 8,20 & - \\
\hline 3 & $3,11(\mathrm{tl})$ & 52,5 & 3,20 & 52,3 \\
\hline 4 & $1,98(\mathrm{dd}, 5,5 ; 2,5)$ & 47,5 & $2,68(\mathrm{dd}, 2,0 ; 5,5)$ & 46,6 \\
\hline 5 & 2,49 & 31,5 & $3,00(\mathrm{~m})$ & 31,0 \\
\hline 6 & - & 150,7 & - & 150,9 \\
\hline 7 & $3,57(\mathrm{~d}, 10,0)$ & 70,1 & $3,61(d, 10,5)$ & 69,4 \\
\hline 8 & $2,69(\mathrm{dd}, 10,0 ; 10,0)$ & 46,1 & 3,20 & 47,5 \\
\hline 9 & - & 53,1 & - & 83,3 \\
\hline 10 & $\begin{array}{c}2,55(\mathrm{dd}, 13,0 ; 8,5) \\
2,81(\mathrm{dd} 13,0 ; 5,0)\end{array}$ & 43,6 & $\begin{array}{c}2,61(\mathrm{dd}, 13,5 ; 7,0) / \\
2,76(\mathrm{dd}, 13,5 ; 5,0)\end{array}$ & 42,8 \\
\hline 11 & $0,39(\mathrm{~d}, 6,5)$ & 12,8 & $0,62(\mathrm{~d}, 7,0)$ & 13,2 \\
\hline 12 & $4,81(\mathrm{~s}) / 5,03(\mathrm{~s})$ & 111,5 & 4,89 (s) / 5,11 (s) & 112,3 \\
\hline 13 & $5,42(\mathrm{dd}, 15,0 ; 10,0)$ & 130,8 & $5,81(\mathrm{dd}, 15,0 ; 10,0)$ & 128,5 \\
\hline 14 & $\begin{array}{c}5,08(\mathrm{ddd}, 15,0 \\
6,0 ; 10,0)\end{array}$ & 131,6 & $\begin{array}{c}5,03(\mathrm{ddd}, 15,0 \\
10,0 ; 3,5)\end{array}$ & $\begin{array}{c}41,6 \\
x\end{array}$ \\
\hline 15 & $\begin{array}{c}1,89(\mathrm{ddl}, 12,5 ; 4,5) / \\
2,15(\mathrm{~m})\end{array}$ & 37,9 & $\begin{array}{l}1,52(\mathrm{~m}) / \\
2,01(\mathrm{~m})\end{array}$ & 41,6 \\
\hline 16 & $2,75(\mathrm{~m})$ & 41,4 & $1,16(\mathrm{~m})$ & 32,9 \\
\hline 17 & - & 209,9 & $0,49(\mathrm{~m}) / 1,64(\mathrm{~m})$ & 34,9 \\
\hline 18 & - & 77,4 & $1,34(\mathrm{~m}) / 1,20(\mathrm{~m})$ & 20,0 \\
\hline 19 & $5,12(\mathrm{dd}, 15,0 ; 2,0)$ & 126,9 & $1,43(\mathrm{~m}) / 1,77(\mathrm{~m})$ & 34,4 \\
\hline 20 & $5,86(\mathrm{dd}, 15,0 ; 2,0)$ & 132,1 & $4,36(\mathrm{~m})$ & 68,6 \\
\hline 21 & $5,26(\mathrm{t}, 2,0)$ & 76,4 & $6,76(\mathrm{dd}, 15,5 ; 4,0)$ & 153,3 \\
\hline 22 & $1,05(\mathrm{~d}, 7,0)$ & 19,2 & $5,70(\mathrm{dd}, 15,5 ; 1,5)$ & 118,1 \\
\hline 23 & $1,38(\mathrm{~s})$ & 24,5 & - & 164,1 \\
\hline 24 & - & 169,9 & $0,82(\mathrm{~d}, 6,5)$ & 20,3 \\
\hline 25 & $2,27(\mathrm{~s})$ & 21,8 & & \\
\hline $1^{\prime}$ & - & 137,1 & - & 137,0 \\
\hline 2’e 6 & $7,15(\mathrm{~d}, 7,0)$ & 129,6 & $7,09(\mathrm{~d}, 7,0)$ & 129,6 \\
\hline 3'e 5 & $7,30(\mathrm{t}, 7,5)$ & 128,3 & $7,26(\mathrm{t}, 7,5)$ & 128,2 \\
\hline $4^{\prime}$ & $7,22(\mathrm{t}, 7,5)$ & 126,4 & $7,17(\mathrm{t}, 7,5)$ & 126,4 \\
\hline
\end{tabular}

*Multiplicidades e constantes de acoplamento $(J)$, em Hz., entre parênteses 
deslocamentos químicos em RMN de ${ }^{1} \mathrm{H},{ }^{13} \mathrm{C}$, constantes de acoplamentos e comparação com dados da literatura ${ }^{8}$. O experimento de rotação ótica forneceu $[\alpha]_{\mathrm{D}}{ }^{25}+71,3$ (c. $\left.0,006 \mathrm{MeOH}\right)$ e permitiu identificar 4 como (+)-citocalasina B. Os valores dos deslocamentos químicos da citocalasina B (4) estão apresentados na Tabela 1 .

A substância 3 foi identificada como 7-decloro-griseofulvina $\left(\mathrm{C}_{17} \mathrm{H}_{18} \mathrm{O}_{6}\right)$ através da análise dos dados espectrométricos e comparação com a literatura ${ }^{12}$. O espectro de massas obtido pela técnica EM-ES (+35 V) apresentou o pico base (100\%) em $\mathrm{m} / \mathrm{z} 319$ $[\mathrm{M}+\mathrm{H}]^{+}$e picos em $m / z, 341[\mathrm{M}+\mathrm{Na}]^{+}$e $m / z, 181\left[\mathrm{M}+\mathrm{C}_{8} \mathrm{H}_{10} \mathrm{O}_{2}\right]^{+}$, indicando 9 graus de insaturação, permitindo propor um sistema de três ciclos e seis insaturações. O espectro de RMN de ${ }^{1} \mathrm{H}$ registrou sinais em $\delta_{\mathrm{H}} 6,15(\mathrm{~d}, J=1,5 \mathrm{~Hz})$ e $\delta_{\mathrm{H}} 5,97(\mathrm{~d}, J=1,5 \mathrm{~Hz})$ sugerindo um padrão de substituição meta, em um anel aromático tetrassubstituído. Observaram-se singletos em $\delta_{\mathrm{H}} 5,47,3,83$, 3,82 e 3,55, indicando a presença de um hidrogênio olefínico e três metoxilas, sendo duas aromáticas e uma sobre sistema insaturado, respectivamente. Foi verificado um dubleto em $\delta_{\mathrm{H}} 0,89$ $(6,5 \mathrm{~Hz})$ e um multipleto em $\delta_{\mathrm{H}} 2,68$, indicativo da presença de uma metila e um hidrogênio metínico. O espectro de RMN de ${ }^{1} \mathrm{H}$ também registrou dois duplos dubletos em $\delta_{\mathrm{H}} 2,33$ (dd, $J=17,0$ e $5,0 \mathrm{~Hz}$ ) e $\delta_{\mathrm{H}} 2,99(\mathrm{dd}, J=17,0$ e $13,0 \mathrm{~Hz})$ com $\delta_{\mathrm{C}} 40,1$, evidenciando tratar-se de um metileno $\alpha$ a um centro estereogênico e a uma carbonila $\alpha, \beta$ insaturada. $\mathrm{O}$ espectro de $\mathrm{RMN}$ de ${ }^{13} \mathrm{C}$ indicou a presença de 17 átomos de carbono, dos quais seis foram atribuídos como sendo do tipo aromático $\left(\delta_{\mathrm{C}} 176,1 ; 170,4 ; 159,1 ; 104,4\right.$; 93,3 e 88,6), dois carbonos $\mathrm{sp}^{2}$ em $\delta_{\mathrm{C}} 171,3$ e 104,8 , dois carbonos carbonílicos em $\delta_{\mathrm{C}} 197,3$ e 192,4, três metoxilas atribuídas aos sinais em $\delta_{\mathrm{C}} 56,6,56,1$ e 56,1 , uma metila em $\delta_{\mathrm{C}} 14,2$, um metino em $\delta_{\mathrm{C}} 36,6$, um carbono metilênico em $\delta_{\mathrm{C}} 40,1$ e um carbono quaternário em $\delta_{\mathrm{C}} 89,9$. Através da análise detalhada do mapa de contorno $g$ HMQC foi possível atribuir os hidrogênios aos respectivos átomos de carbono. Pela análise do mapa de contorno $g \mathrm{HMBC}$ observaram-se as correlações entre $\mathrm{H}-5 \leftrightarrow \mathrm{C}-3 \mathrm{a} / \mathrm{C}$ 7a/C-7/C-6, H-7 $\leftrightarrow \mathrm{C}-3 \mathrm{a} / \mathrm{C}-4 / \mathrm{C}-5 / \mathrm{C}-6,4-\mathrm{OCH}_{3} \leftrightarrow \mathrm{C}-4^{*}$ ou C-6*, $6-\mathrm{OCH}_{3} \leftrightarrow \mathrm{C}-4 *$ ou C-6* (*podem estar trocados), $\mathrm{H}-3^{\prime} \leftrightarrow \mathrm{C}-1^{\prime} /$ $\mathrm{C}-2^{\prime} / \mathrm{C}^{\prime} 4^{\prime}, 2^{\prime} \mathrm{OCH}_{3} \leftrightarrow \mathrm{C}-2^{\prime}, 6^{\prime}-\mathrm{CH}_{3} \leftrightarrow \mathrm{C}^{-} 6^{\prime} / \mathrm{C}-5^{\prime} / \mathrm{C}-1^{\prime}, \mathrm{H}_{\mathrm{a}} 5^{\prime} \leftrightarrow \mathrm{C}-4^{\prime} /$ C-6'/C-1', $\mathrm{H}_{\mathrm{b}}-5^{\prime} \leftrightarrow \mathrm{C}-4^{\prime} / \mathrm{C}-6^{\prime} / \mathrm{CH}_{3}-6^{\prime}$ e $\mathrm{H}-6^{\prime} \leftrightarrow \mathrm{C}-1^{\prime}$, os quais confirmaram 3 como 7-decloro-griseofulvina. A configuração relativa de $\mathbf{3}$ foi determinada por comparação dos valores dos deslocamentos em RMN de ${ }^{1} \mathrm{H},{ }^{13} \mathrm{C}$, constantes de acoplamentos e comparação com dados da literatura ${ }^{12}$. Após experimento de rotação ótica, que forneceu $[\alpha]_{\mathrm{D}}{ }^{25}=+146,1$ (c. 0,0035 em $\mathrm{CHCl}_{3}$ ), foi possível identificar esta substância como (+)-7-declorogriseofulvina. Os valores dos deslocamentos químicos da 7 decloro-griseofulvina (3) estão apresentados na Tabela 2.

Os espectros de $\mathrm{RMN}$ de ${ }^{1} \mathrm{H}$ e ${ }^{13} \mathrm{C}$ de 5 foram comparados com os espectros obtidos para $\mathbf{3}$ e foi possível notar a grande semelhança no perfil espectral destas duas substâncias, sendo a diferença marcante a ausência de um dubleto na região de aromático e a presença de apenas um sinal, na forma de singleto, nesta região $\left(\delta_{\mathrm{H}}\right.$ 6,07). Este fato sugeriu a presença de um anel aromático pentassubstituído em C-7. O espectro de massas de $\mathbf{5}$ obtido pela técnica EM-ES a $+35 \mathrm{~V}$ forneceu os picos $\mathrm{m} / z, 375$, indicando o aduto com sódio $\left([\mathrm{M}+\mathrm{Na}]^{+}\right), m / z 353[\mathrm{M}+\mathrm{H}]^{+}$e $m / z 377[\mathrm{M}+2]^{+}$ evidenciando a presença de um átomo de cloro, sugerindo estar posicionado em C-7. Estas informações aliadas à comparação com a literatura ${ }^{12}$ conduziram à estrutura da griseofulvina $\left(\mathrm{C}_{17} \mathrm{H}_{17} \mathrm{ClO}_{6}\right)$. A rotação ótica de 5 foi medida e o $[\alpha]_{D}=+104$ (c. $0,0035 \mathrm{em}$ acetona) permitiu identificar a substância como (+)-griseofulvina. Os valores dos deslocamentos químicos da griseofulvina (5) estão apresentados na Tabela 2.
Os extratos brutos (PDB e milho) e as substâncias isoladas foram submetidas a um ensaio preliminar para avaliação da atividade antifúngica através da técnica de Bioautografia. O extrato bruto obtido em milho, assim como as substâncias isoladas deste extrato $(\mathbf{3}, 4$ e 5) não se mostraram ativas frente às linhagens Cladosporium cladosporioides e C. sphaerospermum. Entretanto a griseofulvina (5) é atualmente utilizada como um poderoso antimicótico, sendo comercializada em medicamentos como Fulcinâ (Zeneca) e Sporostatin $^{\hat{a}}$ (Shering Plough) com atividade contra várias espécies de Microsporum, Epidermophyton e Trychophyton. O extrato bruto obtido após cultivo de Xylaria sp. em PDB e os compostos ácido 2-hexilideno-3-metilbutanodióico (1) e citocalasina D (2) mostraram-se ativos frente às linhagens $C$. cladosporioides e $C$. spahaerospermum. Os resultados dos limites de detecção das substâncias 1 e 2 estão apresentados na Tabela 3, e este é o primeiro relato do potencial antifúngico demonstrado por estas substâncias.

Tabela 2. Dados de $\mathrm{RMN}$ de ${ }^{1} \mathrm{H}^{*}(500 \mathrm{MHz})$ e ${ }^{13} \mathrm{C}(125 \mathrm{MHz})$ da $7-$ declorogriseofulvina (3) e griseofulvina (5) em $\mathrm{CDCl}_{3}, \delta(\mathrm{ppm})$

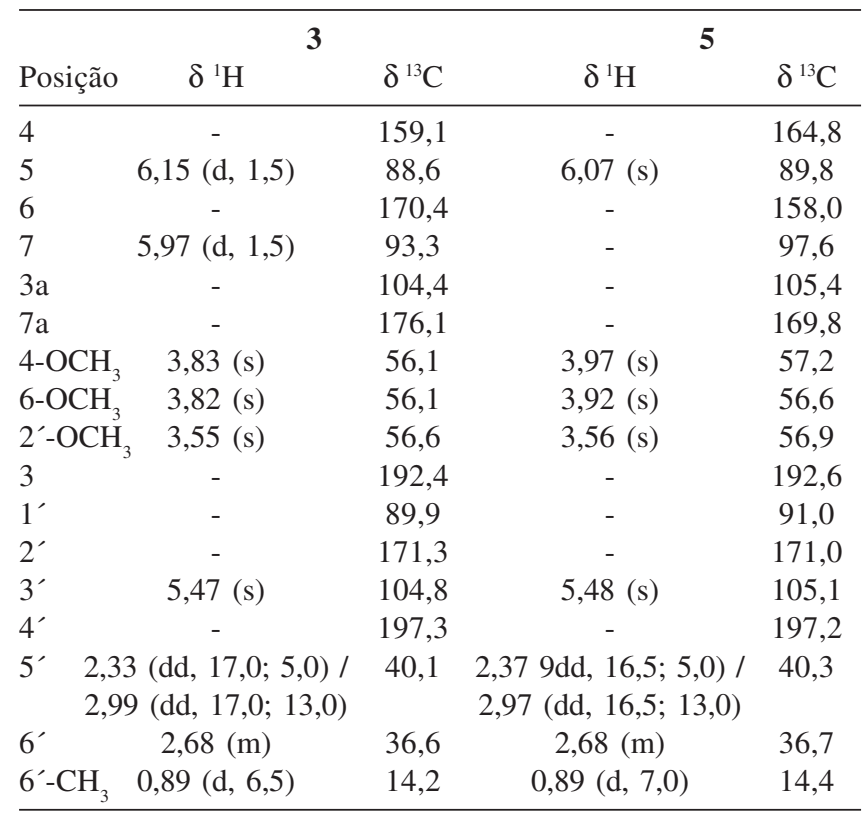

*Multiplicidades e constantes de acoplamento $(J)$, em Hz., entre parênteses.

Tabela 3. Valores dos limites de detecção $(\mu \mathrm{g} / \mathrm{mL})$ obtidos a partir dos bioensaios com as linhagens $C$. cladosporioides e $C$. sphaerospermum

\begin{tabular}{ccc}
\hline Compostos & C. cladosporioides & C. sphaerospermum \\
\hline $\mathbf{1}$ & 25 & 10 \\
$\mathbf{2}$ & 10 & 25 \\
Nistatina & 1 & 1 \\
\hline
\end{tabular}

\section{CONCLUSÃO}

O estudo químico dos extratos produzidos por Xylaria sp. permitiu o isolamento de substâncias com atividade antifúngica em potencial e de interesse comercial, evidenciando os fungos endofíticos como promissores na busca por metabólitos bioativos. Este é o primeiro relato das substâncias $\mathbf{3}, \mathbf{4}$ e $\mathbf{5}$ serem produzidas por um fungo endofítico do gênero Xylaria. As substâncias $\mathbf{1}$ e $\mathbf{2}$ já foram relatadas como metabólitos de Xylaria sp., porém esta é 
a primeira vez como substâncias produzidas por um fungo endofítico.

A produção das substâncias $\mathbf{1}$ e $\mathbf{2}$ com atividade frente aos fungos fitopatogênicos C. cladosporioides e C. sphaerospermum por Xylaria sp. dá indícios de que os endófitos associados com Palicourea marcgravii se encontram em uma relação de simbiose, produzindo substâncias antifúngicas contra possíveis fungos fitopatogênicos.

Os resultados encontrados até o momento evidenciam a potencialidade destes microrganismos e reforçam a necessidade do estudo químico biomonitorado neste nicho de fungos.

\section{AGRADECIMENTOS}

À FAPESP, programa Biota-FAPESP (Instituto Virtual da Biodiversidade, www.biota.org) pelo auxílio financeiro, à CAPES pelas bolsas concedidas a M. C. Cafêu, H. L. Teles e ao CNPq pelas bolsas de G. H. da Silva, V. da S. Bolzani e M. C. M. Young.

\section{REFERÊNCIAS}

1. Demain, A. L.; Biotechnology Advances 2000, 18, 499.

2. Newman, D. J.; Cragg, G. M.; Snader, K. M.; J. Nat. Prod. 2003, 66, 1022.

3. Dreyfuss, M. M.; Chapela, I. H. Em The discovery of natural products with therapeutic potential; Gullo, V. P., ed.; Butterworth-Heinemann: Boston, 1994, p. 49-80.

4. Azevedo, J. L. Em Ecologia microbiana; Melo, I. S.; Azevedo, J. L., eds.; Embrapa-CNPMA: Jaguariúna, 1998, p. 116-137.

5. Maier, W.; Hammer, U.; Dammann, U.; Schulz, B.; Strack, D.; Planta 1997, 202, 36.

6. Fuchs, A.; Homans, A. L.; J. Chromatogr. 1970, 51, 327.

7. Chesters, N. C. J. E.; O’Hagan, D.; J. Chem. Soc., Perkin Trans. 1 1997, 827.

8. Maccotta, A.; Valensin, G.; Gaggelli, N.; Gaggelli, E.; J. Chem. Soc., Perkin Trans. 2 1993, 729.

9. Fujii, Y.; Tani, H.; Ichinoe, M.; Nakajima, H.; J. Nat. Prod. 2000, 63, 132.

10. Konig, G. M.; Wright, A. D.; Aust, H. J.; Drager, S.; Schulz, B.; J. Nat Prod. 1999, 62, 155.

11. Graf, W.; Robert, J. L.; Vederas, J. C.; Tamm, C.; Helv. Chim. Acta 1974, $57,1801$.

12. Sato, Y.; Oda, T.; Tetrahedron Lett. 1976, 44, 3971 